\title{
Asymptomatic pneumomediastinum resulting from air in the epidural space
}

\section{-a case report-}

\author{
Hyun Kyoung Lim ${ }^{1}$, Young Deog Cha ${ }^{1}$, Jang Ho Song ${ }^{1}$, Ji Woong Park ${ }^{1}$, and Mi Hyeon Lee ${ }^{2}$ \\ Department of Anesthesiology and Pain Medicine, ${ }^{1}$ Inha University College of Medicine, Incheon, ${ }^{2}$ Kangnam Sacred Heart Hospital, \\ Hallym University College of Medicine, Seoul, Korea
}

There are no reports regarding pneumomediastinum caused by thoracic epidural block complications. We believe that it is possible to experience an occurrence of pneumomediastinum caused by air in the epidural space after performing a thoracic epidural block using the loss of resistance (LOR) technique with air. We report a witnessed case where pneumomediastinum appeared after a thoracic epidural block. Pneumorrrhachis, paravertebral muscle emphysema, and pneumomediastinum were diagnosed by Positron Emission Tomography-Computed Tomography. Although extremely rare, pneumomediastinum can be caused by an epidural block using LOR technique with air. In order to avoid the above danger, the use of saline or very minimal amount of air is required during a careful LOR technique. (Korean J Anesthesiol 2013; 65: 266-269)

Key Words: Emphysema, Epidural injection, Pneumomediastinum.

An epidural block is performed by placing an epidural needle in the epidural space and injecting local anesthetics. The effectiveness and safety of an epidural block is determined by the proper placement of the epidural needle [1]. Loss of resistance (LOR) technique is the most common method used to determine whether the epidural needle is properly placed in the epidural space. During the LOR technique, air or saline is drawn into a syringe, and continuous or intermittent pressure is applied to the plunger as the epidural needle is advanced toward the epidural space. As gradual pressure is applied, the epidural needle passes through the ligament flavum entering the epidural space where the contents of the syringe are injected due to the sudden loss of resistance. It is generally known that LOR technique with air can increase the incidence of complications such as incomplete analgesia, accidental dural puncture, and postdural puncture headache [2]. However, there have been no reported cases of pneumomediastinum caused by air in the epidural space occurring after an epidural block using the LOR technique with air. The purpose of this case report is to inform doctors to be on alert for the possibility of pneumomediastinum as a complication after the LOR technique with air and to determine a method to reduce this risk.

Received: June 15, 2012. Revised: September 19, 2012. Accepted: October 15, 2012.

Corresponding author: Hyun Kyoung Lim, M.D., Department of Anesthesiology and Pain Medicine, Inha University College of Medicine, 7-206, Sinheung-dong 3-ga, Jung-gu, Incheon 400-711, Korea. Tel: 82-32-890-3968, Fax: 82-32-881-2476, E-mail: hkliman@inha.ac.kr (C) This is an open-access article distributed under the terms of the Creative Commons Attribution Non-Commercial License (http:// creativecommons.org/licenses/by-nc/3.0/), which permits unrestricted non-commercial use, distribution, and reproduction in any medium, provided the original work is properly cited. 


\section{Case Report}

A hospitalized 56-year-old male patient diagnosed with hepatocellular carcinoma was under the care of the Digestive Internal Medical Department. In order to control the patient's pain level, the Digestive Internal Medical Department requested joint treatment from our pain clinic. The patient was $160.5 \mathrm{~cm}$ tall and weighed $54.5 \mathrm{~kg}$. At the time of arrival at our pain clinic, the patient's blood pressure was $145 / 90 \mathrm{mmHg}$ and the heart rate was 68 beats per min (bpm). The patient complained of general weakness and right flank pain. Results of the laboratory examination showed an AST and ALT of 106 and 83, respectively, and no evidence of bleeding. Liver computed tomography (CT) and positron emission tomography (PET) CT taken one week prior revealed a $1-9 \mathrm{~cm}$ sized multiple mass and a right portal vein thrombosis in the liver parenchyma. On a simple chest X-ray, no active lesion was seen. Pulmonary function test (PFT) was not performed as a part of this procedure. The patient's main symptoms were back pain and flank pain limited to the T7 and T8 dermatome level. In order to determine the pain level, epidural block was performed one time at the T7 and T8 level. A consent form was signed by the patient after a detailed explanation of the medical procedure and its possible complications. The epidural block was not performed with the aid of a C-arm guide. In the left decubitus position, $6 \mathrm{ml}$ of $1 \%$ lidocaine was administered as a local anesthesia after betadine was used to cleanse the expansive back region. The paramedian approach was executed using a 22-gauge Tuohy needle at the T7-8 level. Using a Tuohy needle connected to a syringe filled with $5 \mathrm{ml}$ of air, intermittent pressure was applied in order to locate the epidural space using the LOR technique. On the first attempt, approximately at a $5 \mathrm{~cm}$ depth, the lamina of the vertebra was contacted. After the needle was slightly retracted, the needle was advanced towards the cephalad slightly, and at about at $5.5 \mathrm{~cm}$ depth, the LOR was not definitive. Thus, we applied pressure approximately 4 times into the syringe which was fixed at the same depth. After that, one more attempt was made. At the same depth, a slight LOR was continuous, and therefore, we came to the conclusion that it was the epidural space. Following the injection of $8 \mathrm{ml}$ of $0.8 \%$ mepivacaine and $20 \mathrm{mg}$ of triamcinolone, the patient stabilized after being in the supine position for one hour. After one hour, the patient was moved to the ward. His visual analog scale decreased to $3 / 10$ from $7 / 10$. The patient's blood pressure was 130/93 $\mathrm{mmHg}$ and his heart rate was $90 \mathrm{bpm}$. Chest discomfort and dyspnea were not observed and there were no other unusual impressions. Three hours after the patient was transferred to the ward, a PET-CT was performed in order to prepare the patient for Transarterial Chemoembolization (TACE). The results of the PET-CT revealed a new occurrence of pneumomediastinum in addition to the liver mass and portal vein thrombosis observed one week before (Fig. 1A). The position of the pneumomediastinum was extended from T10 (the site where the epidural block was performed) to the C2 epidural space. In addition, air was observed in the paraspinal muscle and epidural space (Fig. 1B). After the patient was informed of the pneumomediastinum, the patient was supplied with oxygen at $2 \mathrm{~L} / \mathrm{min}$ by a nasal cannula and observed closely in the ward. The patient did not have any unusual complaints and was stabilized in bed and a chest CT taken 7 days later showed no signs of pneumomediastinum and pneumothorax.
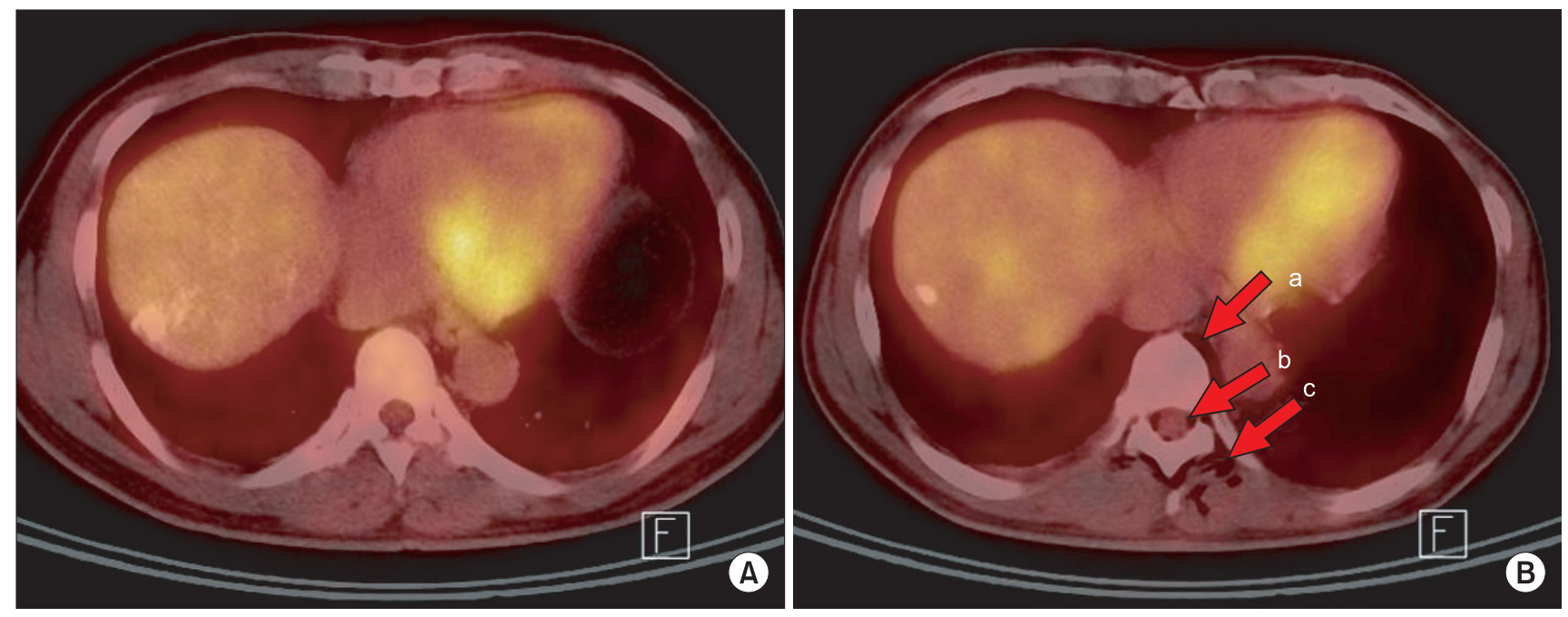

Fig. 1. Pneumomediastinum and pneumothorax after thoracic epidural injection. (A) PET-CT image 1 week before the procedure. There are no signs of pneumomediastinum, epidural air, and paraspinal muscle emphysema. (B) a: Newly developed pneumomediastinum. b: epidural air (pneumorrhachis) c: paraspinal muscle emphysema. 


\section{Discussion}

The main symptom of pneumomediastinum is severe central chest pain. Dyspnea, change in voice and subcutaneous emphysema can also occur along with severe central chest pain or there may be no symptoms at all. Classification of pneumomediastinum depends on the cause of the disease. Spontaneous pneumomediastinum can be caused by infections, asthma, and valsalva maneuvers such as coughing, screaming or vomiting [3]. For the patient in this case, the possibility of spontaneous pneumomediastinum is very slim because, as stated above, a plausible condition did not exist, and one week ago, the PETCT results showed only a liver mass and no other specific conditions. Secondary or acquired pneumomediastinum occurs due to air movement from the airway, lung or bowel to the chest cavity causing physical damage. It can be caused by chest trauma, endotracheal or esophageal procedures, or mechanical ventilation [4]. However, as in this case, secondary pneumomediastinum caused by epidural air following an epidural injection is rarely reported. Pneumorachis is the presence of air in the epidural space or the subarachnoid space. It mainly occurs due to iatrogenic or traumatic causes and occurs frequently after epidural analgesia [5]. In previous reports in which pneumomediastinum and pneumorachis occur simultaneously, pneumomediastinum causing pneumorachis has been reported but not the other way around. It is not exactly known how the air progresses from the posterior mediastinum or retropharyngeal space to the epidural space, but it is presumed that the air freely moves though the neural foramen since there is no fascial barrier [6]. In other words, the air collects in the posterior epidural space because of less resistance from the loose connective tissue compared to the rich vascular network present anteriorly [7]. In our case, there was no evidence of trauma to cause the pneumomediastinum and the epidural needle did not reach the appropriate depth to enter the posterior mediastinum (Fig. 2). Thus, there was little chance of the Tuohy needle to actually penetrate the pleura and reach the posterior mediastinum. From the results of the patient's PET-CT test, at the entry level of the epidural needle, air was confirmed in the back muscle, epidural space and posterior mediastinum, and therefore, it was presumed that the cause of the pneumomediastinum was due to the process of confirming the epidural space with air. During the

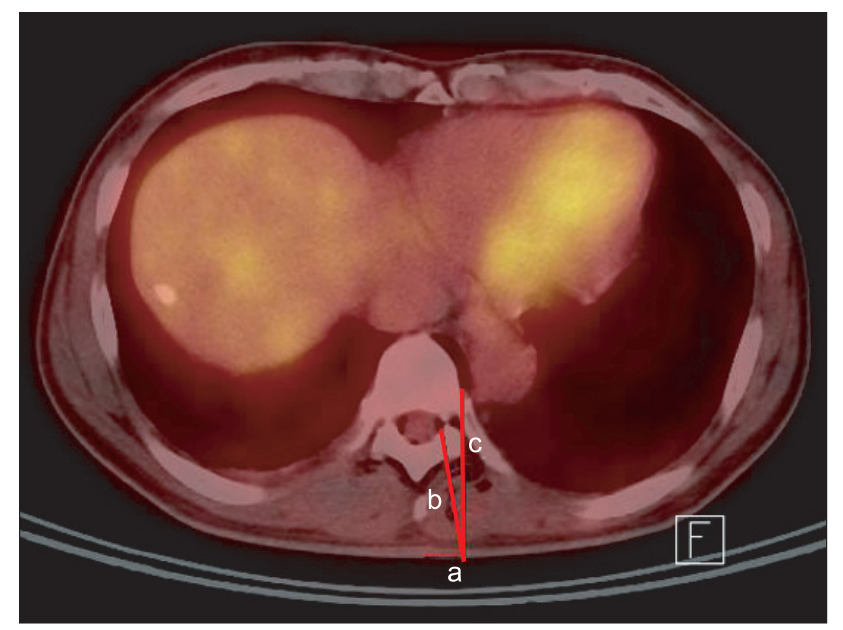

Fig. 2. The relationship between the direction of the needle in an epidural injection and the location of pneumomedaistinum. PETCT image after epidural injection. a: Distance between midline and injection site: $15 \mathrm{~mm}$. b: Distance between injection site and epidural space: $54 \mathrm{~mm}$. c: Distance between injection site and pneumediastinum: $66 \mathrm{~mm}$.

LOR, the individual who performed the procedure, performed LOR at least twice and used approximately $10 \mathrm{ml}$ of air when applying firm pressure. As stated above, from the occurrence of pneumorachis from pneumomediastinum, in a reverse pathway, we can surmise that the air moved from the epidural space to the posterior mediastinum causing pneumomediastinum. This case was an incidental finding without symptoms but the air at the mediastinum had spread from T10 to C2. Therefore, when performing an epidural block, one must keep in mind and beware of the possibility of air in epidural space spreading and moving in an unexpected course.

In conclusion, when performing an epidural block using the LOR technique with air, one must keep in mind the possibility of pneumomediastinum complications, although rare. Therefore, if possible, it is suggested that one should be careful when performing the LOR technique with air and that the quantity of air should be reduced.

\section{Acknowledgments}

This case report was supported by Inha University.

\section{References}

1. Schier R, Guerra D, Aguilar J, Pratt GF, Hernandez M, Boddu K, et al. Epidural space identification: a meta-analysis of complications after air versus liquid as the medium for loss of resistance. Anesth Analg 2009; 109: 2012-21.

2. Shenouda PE, Cunningham BJ. Assessing the superiorty of saline versus air for use in the epidural loss of resistance technique: a literature review. Reg Anesth Pain Med 2003; 28: 48-53. 
3. Chalumeau M, Le Clainche L, Sayeg N, Sannier N, Michel JL, Marianowski R, et al. Spontaneous pneumomediastinum in children. Pediatr Pulmonol 2001; 31: 67-75.

4. Otgün I, Fakioğlu E, Arda IS, Hiçsönmez A. Subcutaneous emphysema and pneumomediastinum due to foreign body aspiration. Eur J Pediatr Surg 2008; 18: 129-30.

5. Oertel MF, Korinth MC, Reinges MH, Krings T, Terbeck S, Gilsbach JM. Pathogenesis, diagnosis and management of pneumorrhachis. Eur Spine J 2006; 15 Suppl 5: 636-43.

6. Drevelengas A, Kalaizoglou I, Petridis A. Pneumorrhachis associated with spontaneous pneumomediastinum. Eur J Radiol 1994; 18: 122-3.

7. Bernaerts A, Verniest T, Vanhoenacker F, Van den Brande P, Petré C, De Schepper AM. Pneumomediastinum and epidural pneumoatosis after inhalation of "Ecstasy". Eur Radiol 2003; 13: 642-3. 